\title{
UTILIZAÇÃO DE SERVIÇOS DE SAÚDE RELACIONADOS COM A INFECÇÃO VIH POR PARTE DE HOMENS GAYS: ACESSO A INFORMAÇÃO, PREVENÇÃO E TESTE
}

\author{
Sónia Dias ${ }^{12}$ \\ Ana Gama ${ }^{2} 3$ \\ Ricardo Fuertes ${ }^{3}$ \\ Gabriela Cohen ${ }^{3}$ \\ Ricardo Rosa ${ }^{4}$ \\ Luís Mendão ${ }^{3}$
}

Resumo: O estudo tem como objectivo descrever a utilização de serviços de saúde para informação, prevenção e teste do VIH por parte de homens gays em Portugal e identificar factores associados a ter efectuado o teste VIH em diferentes serviços.

Para este estudo de abordagem participativa foi aplicado um inquérito por questionário a 1046 homens gays. Cerca de $27 \%$ dos participantes refere que recorreu ao CAD para obter informação sobre VIH/sida. Cerca de $83 \%$ recebeu gratuitamente preservativos no último ano. A maioria (88\%) já efectuou o teste do VIH. Destes, $74 \%$ realizou-o no SNS, $22.5 \%$ num serviço privado e $6.5 \%$ numa ONG, verificando-se diferenças significativas quanto à idade, escolaridade, rendimento e nacionalidade. Os resultados indicam uma utilização dos serviços de saúde para informação, prevenção e teste do VIH pela maioria dos participantes. No entanto, é importante continuar a desenvolver esforços para promover a utilização dos serviços, em especial pelos grupos desta população que se encontram em maior vulnerabilidade, trabalhando em proximidade com as comunidades.

Palavras-chave: homens gays; utilização dos serviços de saúde; teste do VIH.

\footnotetext{
${ }^{1}$ Autor para correspondência: Sónia Dias, Instituto de Higiene e Medicina Tropical, Rua da Junqueira, 100, 1349-008 Lisboa, Tel: 21 3652600, Fax: 21 3632105, e-mail: sfdias@ihmt.unl.pt,smfdias@yahoo.com

2 Instituto de Higiene e Medicina Tropical, CMDT/IHMT, Universidade Nova de Lisboa

${ }^{3}$ GAT - Grupo Português de Actividades sobre Tratamentos de VIH/SIDA

${ }^{4}$ Associação para o Desenvolvimento da Medicina Tropical
} 
Utilization of HIV health services by gay men: Access to information, prevention and testing (Abstract): This study aims to describe utilization of health services for HIV information prevention and testing by gay men in Portugal and identify factors associated with having been tested for HIV in different settings. In this study based on a participatory approach a questionnaire was applied to 1046 gay men. About $27 \%$ of participants referred to have used CAD to obtain information about HIV/Aids. Around $83 \%$ received free condoms during last year. The majority $(88 \%)$ have been tested. Among these, $74 \%$ had a test in the NHS, $22.5 \%$ in a private service and $6.5 \%$ in an NGO, with significant differences regarding age, education, income and nationality. The results point to a utilization of health services for HIV information, prevention and testing among most of participants. Nevertheless, it is important to undertake efforts continuously to promote health services utilization, in particular by groups of gay men population that are in greater vulnerability, working closely with communities.

Key Words: gay men; utilization of health services; HIV testing.

\section{Introdução}

Desde o início da epidemia nos anos 80, os homens homossexuais, bissexuais e outros homens com identidades e orientações sexuais diversas que têm relações sexuais com homens, designados desde 1994 pelo termo mais abrangente de homens que têm sexo com homens (HSH) (Young \& Meyer, 2005), têm sido desproporcionalmente afectados pelo VIH (WHO, 2011). A infecção pelo VIH entre os homens que têm sexo com homens permanece num elevado número de países, como os da Europa Ocidental, EUA e Austrália, da América Central e do Sul e do Sudoeste Asiático, verificando-se nos últimos anos um aumento da prevalência nestas populações (Likatavicius et al., 2008; UNAIDS, 2010a; van de Laar, 2009; van Griensvena et al., 2009). Mesmo em regiões e países em que a infecção é do tipo generalizado (a prevalência na população geral com idade entre os 15 e 49 anos é superior a 1\%), os homens que têm sexo com homens tendem a ser mais afectados pelo VIH do que a população geral (Baral et al., 2007).

Dados internacionais estimam que, dos casos de VIH reportados em 2009 nos países da Região Europeia da OMS, cerca de 18\% referem-se a transmissão homossexual masculina, e que o número de casos diagnosticados nesta população aumentou em 27\% entre 2004 e 2009, taxa que é superior à do aumento da infecção por via heterossexual ou relacionada com a utilização de drogas injectáveis (ECDC/WHO, 2010).

Os dados relativos à infecção pelo VIH/sida em Portugal indicam que, em 2009, este era um dos países da União Europeia com maior incidência de infecção (9.9 diagnósticos por 100.000 pessoas) e maior prevalência do VIH 
(0.6\% entre os indivíduos do grupo etário dos 15-49 anos) (ECDC/WHO, 2010; UNAIDS, 2010a). As principais vias de transmissão estão associadas à transmissão sexual (heterossexual e homossexual masculina) e à utilização de drogas injectáveis (Coordenação Nacional para a Infecção VIH/sida, 2007; INSA, 2011). A epidemia do VIH/sida em Portugal é do tipo concentrado, observando-se prevalências superiores a 5\% em alguns grupos específicos como os utilizadores de drogas injectáveis e os reclusos (Coordenação Nacional para a Infecção VIH/sida, 2007). A informação sobre a infecção VIH nos homens que têm sexo com homens é escassa. No entanto, dados recentes estimam que Portugal é o terceiro país europeu com maior número de novos casos diagnosticados atribuídos a transmissão sexual entre homens, a seguir à Inglaterra e à Holanda e a par da Bélgica (ECDC/WHO, 2010), e que esta via de transmissão representa cerca de $21 \%$ do total de novos casos diagnosticados em 2010 (INSA, 2011).

Neste contexto, os homens que têm sexo com homens têm vindo a ser considerados particularmente vulneráveis à infecção pelo VIH, constituindo-se como um dos alvos prioritários de intervenção no âmbito do Programa Nacional de Prevenção e Controlo da Infecção VIH/sida 2007-2010 (Coordenação Nacional para a Infecção VIH/sida, 2007).

Diversos factores têm sido associados a uma maior vulnerabilidade dos homens que têm sexo com homens à infecção pelo VIH (AMFAR, 2006). À partida existe uma vulnerabilidade biológica na medida em que o sexo anal (homossexual ou heterossexual) desprotegido implica maior risco de transmissão do que o sexo vaginal (UNAIDS, 2009a).

A nível comportamental, estudos indicam que persistem práticas sexuais desprotegidas (Chen et al., 2002; Koblin et al., 2006) que envolvem um elevado risco de transmissão de VIH devido à elevada concentração epidemiológica (AMFAR, 2008).

Adicionalmente, factores sociais e estruturais, como a discriminação, a marginalização e a homofobia, presentes em maior ou menor medida na generalidade dos países, e muitas vezes manifestados pela existência de leis que criminalizam comportamentos homossexuais (ainda existentes em 76 países) (Ottosson, 2010), têm sido associados a um maior risco de infecção pelo VIH (Mayer et al., 2008; Valdisserri, 2002). Num contexto social de discriminação com base na orientação sexual, pessoas com identidades sexuais ou de género estigmatizadas tendem a apresentar elevadas prevalências de experiências de violência, de distúrbios de ansiedade e depressão, e de consumo de substâncias psicoactivas como estratégia de coping (Koblin et al., 2006; Mayer et al., 2008; Meyer, 2003; Reisner et al., 2009; Stall et al., 2003, Wolitski \& Fenton, 2011). Nestas situações, os indivíduos podem ter limitada capacidade e motivação para adoptar medidas protectoras da infecção, como o uso consistente do preservativo (Hatzenbuehler et al., 2011). 
Por outro lado, o estigma e a discriminação dificultam o acesso à informação específica em saúde, à prevenção (por exemplo, à obtenção de preservativos ou gel lubrificante), ao tratamento e aos cuidados de saúde (WHO, 2009). Efectivamente, estudos indicam que as populações LGBT, como os homens homossexuais e bissexuais, tendem a nem sempre utilizar os serviços disponíveis como consequência de experiências negativas vivenciadas nos serviços de saúde, associadas a estigmatização e dupla discriminação, quer com base na orientação sexual, quer associada ao VIH/sida, e por não se sentirem confortáveis para revelar a sua orientação sexual aos profissionais de saúde (Eliason \& Schope, 2001; Mimiaga et al., 2009; Schuster et al., 2005; Stein \& Bonuck, 2001).

De forma global, a utilização dos serviços de saúde é reconhecida como uma importante condição para a obtenção de ganhos em saúde (WHO, 2007). No que diz respeito ao $\mathrm{VIH} /$ sida, o acesso à informação, rastreio e cuidados de saúde é fundamental para garantir o acesso atempado a tratamentos que permitam o controlo de uma doença mortal e reduzir o número de novas transmissões (Heymer \& Wilson, 2011; UNAIDS, 2010a; WHO, 2011). De forma a desenvolver e implementar intervenções adequadas torna-se necessário delinear estratégias de acção adaptadas à diversidade sexual, às especificidades e às necessidades em saúde desta população. Neste sentido é crucial alargar o conhecimento sobre a procura de cuidados de saúde e a utilização dos serviços por parte dos homens gays em Portugal, e em especial no contexto do VIH/sida. Apesar da escassa investigação em Portugal na área do VIH/sida com estas populações, um maior conhecimento desta temática pode contribuir para a definição de estratégias de intervenção no quadro da promoção da utilização dos serviços de saúde, que visem reduzir a vulnerabilidade destes grupos (Bakker et al., 2006; Dowshen et al., 2009).

O presente estudo tem como objectivo descrever a utilização de serviços de saúde para informação, prevenção e teste do VIH por parte de homens gays em Portugal, bem como identificar factores associados ao ter efectuado o teste VIH em diferentes serviços. Desta forma pretende-se que o conhecimento adquirido possa contribuir para o desenvolvimento de estratégias e políticas de prevenção adequadas às necessidades específicas das populações.

\section{Métodos}

No âmbito de um projecto para caracterizar os conhecimentos, atitudes e práticas relacionadas com o VIH/sida em homens que têm sexo com homens foi aplicado um inquérito por questionário a homens que reportaram já ter tido relações sexuais com outros homens independentemente de como se identificavam quanto à sua orientação sexual. $\mathrm{O}$ presente artigo incide nos 
dados referentes aos participantes que reportaram ser homossexuais ou bissexuais na resposta à pergunta "Como descreve a sua orientação sexual?", tendo sido excluídos os participantes que indicaram ser heterossexuais.

\section{Participantes}

Um total de 1046 homens homossexuais e bissexuais responderam ao questionário. A recolha de dados decorreu entre Dezembro de 2009 e Agosto de 2010 em locais de socialização, associações LGBT e eventos gay de várias regiões do país (Grande Porto, Grande Lisboa, Centro e Sul do país).

$\mathrm{O}$ estudo foi desenvolvido com base numa abordagem participativa, tendo sido estabelecidas parcerias com associações e organizações da sociedade civil que trabalham com as populações LGBT, bem como com elementos da população homossexual masculina e outros homens que têm sexo com homens, no sentido de envolver os diversos membros da comunidade nas diferentes etapas do projecto. Mais especificamente, os vários parceiros participaram activamente na concepção e delineamento do projecto, na elaboração dos instrumentos de recolha de dados, no recrutamento e treino de inquiridores, na organização e execução do trabalho de terreno e na interpretação e divulgação dos resultados. Em termos operacionais, de forma a envolver os parceiros no planeamento e implementação das várias actividades foi constituído um Conselho Consultivo da Sociedade Civil e das Comunidades HSH, composto por colectivos LGBT e líderes/activistas.

Neste estudo utilizou-se o método de amostragem por bola de neve. Numa primeira fase recorreu-se aos contactos disponibilizados da rede social das organizações comunitárias parceiras para identificar potenciais participantes, atendendo aos critérios de inclusão no estudo: ter idade igual ou superior a 18 anos e reportar ter tido pelo menos uma relação sexual com outro homem nos últimos 12 meses. Aos participantes que responderam ao questionário foi-lhes proposto convidar outros homens das suas redes sociais virtuais e não virtuais para participarem no estudo que, por sua vez, recrutaram outros homens, num processo semelhante a uma bola de neve (Wasserman et al., 2005). Este método de amostragem, apesar de não aleatório, tem sido reconhecido como eficaz no estudo de populações de difícil acesso, como as populações LGBT (Faugier \& Sargeant, 1997; Morrison, 1988). A utilização desta técnica permite que se obtenha um grupo de participantes suficientemente numeroso e diversificado num período de tempo reduzido. Outra vantagem desta técnica é a confiança que se estabelece entre os potenciais participantes, o que pode aumentar a adesão dos indivíduos do grupo à participação no estudo (Sadler et al., 2010). 


\section{Instrumento}

O instrumento de recolha de dados foi construído em colaboração com os parceiros do estudo, com base nos indicadores desenvolvidos pela Sessão Especial da Assembleia Geral das Nações Unidas sobre VIH/sida (UNGASS), Organização Mundial de Saúde (OMS) e Centro Europeu para a Prevenção e Controlo das Doenças (ECDC). Estes indicadores, alguns dos quais específicos para determinadas populações como os homens gays e outros homens que têm sexo com homens, foram desenvolvidos de forma a criar dados comparáveis a nível internacional (UNAIDS, 2009b). O questionário desenvolvido para este estudo incluiu questões de resposta fechada sobre características sociodemográficas (especificamente idade, habilitações literárias, rendimento mensal do agregado familiar, nacionalidade e situação administrativa), orientação sexual, conhecimentos e atitudes face ao VIH, práticas sexuais, consumo de substâncias psicoactivas, teste do VIH e acesso e utilização de serviços de saúde. Neste artigo são apresentados os resultados sobre a utilização dos serviços de saúde para informação, prevenção e teste do VIH.

\section{Procedimentos}

Os questionários foram aplicados por entrevista individual por inquiridores treinados. Neste sentido, numa fase inicial, as organizações parceiras no projecto indicaram indivíduos, geralmente pares do grupo em estudo, a fim de desempenharem a função de inquiridor. Posteriormente, os indivíduos seleccionados receberam formação específica sobre técnicas gerais de aplicação e preenchimento do questionário e sobre o procedimento de recolha de dados. A opção pela aplicação do questionário por inquiridores visou garantir a uniformidade de procedimentos do processo de recolha de dados, assegurar uma maior qualidade da informação recolhida e permitir incluir participantes com potenciais dificuldades ao nível da compreensão escrita.

O consentimento informado oral dos participantes foi obtido e, de forma a manter o seu anonimato, foi assinado pelos inquiridores, atestando a autorização e a confidencialidade da participação no estudo (Ordem dos Psicólogos Portugueses, 2011).

\section{Análise de dados}

Os questionários preenchidos foram submetidos a um processo de leitura óptica. Posteriormente, os dados foram inseridos numa base de dados do programa estatístico IBM SPSS 19. Para a análise dos dados utilizou-se o teste Qui-quadrado de Pearson (com um nível de significância de .05), de forma a analisar a associação entre características sociodemográficas e o ter realizado o teste do VIH no SNS, num serviço de saúde privado e numa ONG. 


\section{Resultados}

\section{Caracterização sociodemográfica}

Do total de participantes, $42.9 \%$ tem idade entre 21 e 30 anos e $30.8 \%$ entre 31 e 40 anos (Tabela 1). Os níveis de escolaridade mais frequentes são o ensino superior (licenciatura, pós-licenciatura, mestrado ou doutoramento) (39.6\% dos inquiridos) e o ensino secundário (39.3\%). A maior parte dos participantes declara que o rendimento mensal do agregado familiar é de entre $501 €$ e $2000 €$ (1001€-2000€: 37.8\%; 501€-1000€: 33.3\%). A grande maioria dos inquiridos tem nacionalidade portuguesa $(90.3 \%)$. Dos participantes com nacionalidade estrangeira $(9.7 \%)$, a maior parte encontra-se em situação regular em Portugal (87.5\%).

\section{Utilização dos serviços saúde para informação e prevenção do VIH}

Do total de participantes, $55.7 \%(\mathrm{n}=574)$ declara ter recorrido ao Serviço Nacional de Saúde (SNS) há menos de 6 meses, 25.1\% $(\mathrm{n}=258)$ recorreu há mais de 6 meses e menos de um ano e 19\% $(n=195)$ há mais de um ano (dados não apresentados em tabela). Cerca de $38 \%$ dos participantes considera o atendimento no SNS como aceitável, $37.5 \%$ como bom, $12.3 \%$ como muito bom e $12.2 \%$ como mau (dados não apresentados em tabela).

Aproximadamente $32 \%$ dos participantes reporta nunca ter recorrido a um serviço para obter informação sobre VIH/sida. Dos que reportam já ter recorrido, cerca de $27 \%$ recorreu a Centros de Aconselhamento e Detecção (CAD), $17.6 \%$ a um hospital público e $16.7 \%$ a um centro de saúde para obter esse tipo de informação (Tabela 2).

A principal fonte a que os participantes recorrem para obter informação sobre VIH/sida é a Internet (52\%), seguido dos médicos e profissionais de saúde (20.5\%), e da comunicação social (11.2\%) (Tabela 2). Apenas $2.3 \%$ dos inquiridos refere nunca ter tentado obter informação sobre VIH/sida e $1.2 \%$ considera não necessitar desse tipo de informação.

Mais de metade dos participantes $(61.4 \%, \mathrm{n}=626)$ afirma não ter sido abrangido por nenhuma campanha de prevenção do VIH/sida nos últimos 12 meses (Tabela 2). Cerca de $83 \%(n=862)$ dos inquiridos refere ter recebido gratuitamente preservativos no mesmo período (Tabela 2). 
Tabela 1: Características sociodemográficas dos participantes.

\begin{tabular}{|c|c|c|}
\hline & $\mathbf{n}$ & $\%$ \\
\hline \multicolumn{3}{|l|}{ Idade (anos) $(n=1045)$} \\
\hline $18-20$ & 95 & 9.1 \\
\hline $21-30$ & 448 & 42.9 \\
\hline $31-40$ & 322 & 30.8 \\
\hline $41-50$ & 133 & 12.7 \\
\hline$\geq 51$ & 47 & 4.5 \\
\hline \multicolumn{3}{|c|}{ Habilitações literárias $(n=1035)$} \\
\hline$\leq 1^{\circ}$ ciclo & 15 & 1.5 \\
\hline $2^{\circ} / 3^{\circ}$ ciclo & 203 & 19.6 \\
\hline Ensino Secundário & 407 & 39.3 \\
\hline Ensino Superior & 410 & 39.6 \\
\hline \multicolumn{3}{|c|}{$\begin{array}{l}\text { Rendimento mensal do agregado familiar } \\
(n=1017)\end{array}$} \\
\hline$\leq 500 €$ & 88 & 8.6 \\
\hline $501 €-1000 €$ & 339 & 33.3 \\
\hline $1001 €-2000 €$ & 384 & 37.8 \\
\hline$\geq 2001 €$ & 206 & 20.3 \\
\hline \multicolumn{3}{|c|}{ Nacionalidade $(n=1032)$} \\
\hline Portuguesa & 932 & 90.3 \\
\hline Estrangeira & 100 & 9.7 \\
\hline \multicolumn{3}{|c|}{ Situação administrativa $(n=88)$} \\
\hline Situação Regular & 77 & 87.5 \\
\hline Situação Irregular & 11 & 12.5 \\
\hline
\end{tabular}


Tabela 2: Serviços utilizados para obter informação sobre VIH/sida, principal fonte de informação e participação em campanhas e iniciativas de prevenção no último ano.

\begin{tabular}{lrr}
\hline & n & \% \\
\hline $\begin{array}{l}\text { Serviços a que já recorreu para obter informação } \\
\text { sobre VIH/sida (n=1046) }\end{array}$ & & \\
Centro de Saúde & 175 & 16.7 \\
Hospital Público & 184 & 17.6 \\
Clínica/Hospital Privado & 44 & 4.2 \\
Farmácia & 15 & 1.4 \\
Linha SIDA/serviços telefónicos & 49 & 4.7 \\
CAD & 285 & 27.2 \\
CNSIDA & 24 & 2.3 \\
ONG & 118 & 11.3 \\
Outro & 148 & 14.1 \\
Nenhum & 332 & 31.7 \\
\hline Principal fonte de informação a que já recorreu para & & \\
obter informação sobre VIH/sida (n=973) & 199 & 20.5 \\
Médicos/Profissionais de Saúde & 109 & 11.2 \\
Comunicação Social & 506 & 52.0 \\
Internet & 63 & 6.5 \\
Amigos e Parentes & 13 & 1.3 \\
Professores & 26 & 2.7 \\
ONG & 2 & 0.2 \\
Farmacêutico & 20 & 2.0 \\
Outro & 22 & 2.3 \\
Nunca tentou obter informação & 1 & 0.1 \\
Não consegue obter informação & 12 & 1.2 \\
Não precisa de informação & 626 & 61.4 \\
\hline Foi abrangido por campanhas de prevenção do & 38.6 \\
Sim & & \\
Não & & \\
\hline Recebeu preservativos gratuitos nos últimos 12 meses? & & \\
(n=1043) & & \\
Sim & & \\
Não & & \\
\hline & & \\
\hline
\end{tabular}


$\mathrm{Na}$ eventualidade de poderem estar infectados, os inquiridos referem que recorreriam principalmente ao $\mathrm{CAD}(44.7 \%)$, a um hospital público (36.3\%) e ao centro de saúde (28.1\%) (Tabela 3). Apenas 3.1\% dos inquiridos reporta não saber o que fazer nessa situação. No caso dos inquiridos que já suspeitaram estar infectados, $27.2 \%$ refere que recorreu ao CAD, 16.6\% recorreu ao hospital público e $13.3 \%$ ao centro de saúde.

Tabela 3: Locais onde os inquiridos iriam e onde já recorreram por motivo de suspeita de infecção pelo VIH.

\begin{tabular}{lrr}
\hline & $\mathbf{n}$ & \% \\
\hline $\begin{array}{l}\text { Se suspeitasse estar infectado, onde recorreria? } \\
\text { (n=1046) }\end{array}$ & & \\
Centro de Saúde & 294 & 28.1 \\
Hospital Público & 380 & 36.3 \\
Clínica/Hospital Privado & 117 & 11.2 \\
Farmácia & 9 & 0.9 \\
CAD & 468 & 44.7 \\
ONG & 95 & 9.1 \\
Linha SIDA & 130 & 12.4 \\
Não saberia o que fazer & 32 & 3.1 \\
Outro & 55 & 5.3 \\
\hline Se já suspeitou estar infectado, onde recorreu? \\
(n=1046)
\end{tabular}




\section{Teste do VIH}

Do total de participantes, $95.7 \%(n=999)$ sabe que o teste do VIH pode ser efectuado de forma gratuita e confidencial (Tabela 4). A maioria $(88 \%)$ afirma ter realizado o teste VIH pelo menos uma vez na vida e $69.9 \%$ nos últimos 12 meses. Aproximadamente $74 \%$ dos participantes reportou já ter efectuado o teste no SNS, $22.5 \%$ numa clínica, laboratório ou hospital privado e $6.5 \%$ numa organização não-governamental $(\mathrm{ONG})$ ou unidade móvel.

Tabela 4. Conhecimento sobre as condições para realizar o teste do VIH, realização do teste e local.

\begin{tabular}{lrr}
\hline & n & \% \\
\hline O teste do VIH pode ser gratuito e confidencial? (n=1044) & & \\
Sim & 999 & 95.7 \\
Não & 31 & 3.0 \\
Não sabe & 14 & 1.3 \\
\hline Já alguma vez fez o teste do VIH? (n=1043) & & \\
Sim & 918 & 88.0 \\
Não & 122 & 11.7 \\
Não responde & 3 & 0.3 \\
\hline Onde efectuou o teste? (n=918) & 60 & 6.5 \\
ONG/Unidade móvel & 678 & 73.9 \\
Serviço Nacional de Saúde (SNS) & 207 & 22.5 \\
Clínica/Laboratório/Hospital Privado & 19 & 2.1 \\
Outro & & \\
\hline
\end{tabular}

Na Tabela 5 apresentam-se os resultados relativos à realização do teste VIH pelo menos uma vez na vida nos diferentes locais de acordo com as características sociodemográficas dos inquiridos.

Os participantes que realizaram o teste VIH no SNS reportam mais frequentemente ter escolaridade equivalente ou inferior ao ensino secundário $\left(\chi^{2}=25.609, g l=3, p<.001\right)$, e um rendimento mensal inferior a $1000 €\left(\chi^{2}\right.$ $=13.254, g l=3, p=.004)$, em comparação com os que não efectuaram o teste no SNS.

Entre os inquiridos que efectuaram o teste em serviços privados (clínica, laboratório ou hospital privado), uma maior proporção tem mais de 31 
anos $\left(\chi^{2}=13.091, g l=4, p=.011\right)$, tem escolaridade de nível superior $\left(\chi^{2}=\right.$ 33.093, $g l=3, p<.001)$ e declara ter um rendimento mensal superior a $1000 €\left(\chi^{2}=23.938, g l=3, p<.001\right)$, comparativamente aos participantes que reportam nunca ter realizado o teste VIH num serviço de saúde privado.

Tabela 5. Factores sociodemográficos associados à realização do teste VIH no SNS, num serviço privado e numa ONG.

\begin{tabular}{|c|c|c|c|c|c|c|c|c|c|}
\hline & \multicolumn{9}{|c|}{ Locais de realização do teste VIH } \\
\hline & \multicolumn{2}{|c|}{ SNS } & \multicolumn{3}{|c|}{ Privado } & \multicolumn{4}{|c|}{ ONG } \\
\hline & $\begin{array}{c}\text { Sim } \\
\%\end{array}$ & $\begin{array}{c}\text { Não } \\
\%\end{array}$ & $p$ & $\begin{array}{c}\text { Sim } \\
\%\end{array}$ & $\begin{array}{c}\text { Não } \\
\%\end{array}$ & $p$ & $\begin{array}{c}\text { Sim } \\
\%\end{array}$ & $\begin{array}{c}\text { Não } \\
\%\end{array}$ & $p$ \\
\hline Total $(n=918)$ & 73.9 & 26.1 & & 22.5 & 77.5 & & 6.5 & 93.5 & \\
\hline \multicolumn{10}{|l|}{$\begin{array}{l}\text { Idade (anos) } \\
(\mathrm{n}=917)\end{array}$} \\
\hline $18-20$ & 7.1 & 5.9 & .236 & 3.5 & 7.7 & $.011 *$ & 15.0 & 6.2 & $.050^{*}$ \\
\hline $21-30$ & 42.9 & 36.8 & & 35.9 & 42.9 & & 45.0 & 41.1 & \\
\hline $31-40$ & 33.3 & 34.7 & & 35.9 & 33.1 & & 28.3 & 34.1 & \\
\hline $41-50$ & 12.4 & 17.2 & & 18.9 & 12.1 & & 6.7 & 14.1 & \\
\hline$\geq 51$ & 4.3 & 5.4 & & 5.8 & 4.2 & & 5.0 & 4.5 & \\
\hline
\end{tabular}

\section{Habilitações}

literárias $(\mathrm{n}=\mathbf{9 0 8})$

$\leq 1^{\circ}$ ciclo

$1.4 \quad 1.7<.001 * 0.5$

$1.9<.001 *$

$6.7 \quad 1.2<.001^{*}$

$2^{\circ} / 3^{\circ}$ ciclo

19.616 .8

$13.7 \quad 20.3$

$31.7 \quad 17.9$

Ensino Secundário $\quad 42.4 \quad 26.9$

$27.3 \quad 41.5$

$33.3 \quad 38.7$

Ensino Superior

$36.6 \quad 54.6$

$58.5 \quad 36.3$

$28.3 \quad 42.2$

\begin{tabular}{lrrlrrrrrr}
\hline $\begin{array}{l}\text { Rendimento } \\
\text { mensal (n=896) }\end{array}$ & & & & & & & & & \\
$\leq 500 €$ & 9.2 & 6.9 & $.004 *$ & 6.4 & 9.2 & $<.001 *$ & 13.8 & 8.3 & .171 \\
$501 €-1000 €$ & 35.8 & 26.5 & & 22.3 & 36.6 & & 41.4 & 32.8 & \\
$1001 €-2000 €$ & 37.3 & 39.7 & & 41.6 & 36.9 & & 29.3 & 38.5 & \\
$\geq 2001 €$ & 17.7 & 26.9 & & 29.7 & 17.3 & & 15.5 & 20.4 & \\
\hline $\begin{array}{l}\text { Nacionalidade } \\
(\mathbf{n}=\mathbf{9 0 4})\end{array}$ & & & & & & & & & \\
Portuguesa & 91.0 & 87.3 & .106 & 89.6 & 90.2 & .813 & 81.4 & 90.7 & $.021^{*}$ \\
Estrangeira & 9.0 & 12.7 & & 10.4 & 9.8 & & 18.6 & 9.3 & \\
\hline
\end{tabular}

\section{Situação adminis-}

trativa $(n=79)$

$\begin{array}{llllllllll}\text { Situação Regular } & 90.4 & 85.2 & .490 & 83.3 & 90.2 & .423 & 81.8 & 89.7 & .445\end{array}$

Situação Irregular $\quad 9.6 \quad 14.8$

* Diferença significante para $p<.05$. 
Dos participantes que realizaram o teste VIH numa ONG ou unidade móvel, uma maior proporção tem menos de 31 anos e mais de 51 anos de idade $\left(\chi^{2}=9.512, g l=4, p=.050\right)$, tem escolaridade igual ou inferior ao $3^{\circ}$ ciclo $\left(\chi^{2}=19.596, g l=3, p<.001\right)$ e tem nacionalidade estrangeira $\left(\chi^{2}=\right.$ $5.315, g l=1, p=.021$ ), quando comparado com os participantes que reportam nunca ter feito o teste VIH numa ONG.

\section{Discussão}

Neste estudo pretendeu-se descrever a utilização de serviços de saúde para informação, prevenção e teste do VIH por parte de homens gays em Portugal. Dada a limitada informação existente sobre a temática em Portugal, este estudo pode contribuir para um conhecimento que será pertinente para o desenvolvimento de estratégias que possam tornar os serviços de saúde mais adequados às necessidades das diversas populações e eficazes em termos de prevenção do VIH/sida.

No que respeita à procura de informação sobre VIH/sida, a maior parte dos participantes já recorreu a serviços de saúde, nomeadamente ao CAD, ao hospital público ou ao centro de saúde. Não obstante, dado que cerca de um terço dos participantes reportou nunca ter procurado obter informação sobre VIH/sida em qualquer tipo de serviço, apesar de poder dever-se a diferentes razões, pode-se especular sobre a existência de potenciais dificuldades no acesso aos serviços de saúde por uma parte desta população. No presente estudo não se obteve informação que permitisse averiguar a adequação da informação veiculada pelos serviços gerais às necessidades específicas de pessoas com práticas sexuais minoritárias. Também não foram exploradas as questões sobre as barreiras no acesso e utilização dos serviços de saúde, pelo que futuras investigações nestas áreas seriam pertinentes. No entanto, várias investigações apontam no sentido de que grupos LGBT experienciam muitas vezes dificuldades em recorrer aos serviços associadas a estigma e discriminação (Clark et al., 2001; Melendez \& Pinto, 2009; Schuster et al., 2005). Neste contexto, os indivíduos podem optar por utilizar outros meios de informação alternativos para obtenção de informação sobre VIH/sida, o que de facto é sugerido pelos dados do presente estudo, que indicam que para mais de metade da população inquirida a internet é a principal fonte de informação sobre VIH/sida a que recorrem. Além da redução de potenciais barreiras no acesso aos serviços de saúde e da promoção da sua utilização, pode ser pertinente desenvolver programas de informação sobre $\mathrm{VIH} /$ sida com recurso aos meios de comunicação virtual.

A grande maioria dos inquiridos afirmou que recebeu preservativos gratuitos no último ano. Estes resultados são interessantes pois podem indi- 
car que os esforços até agora desenvolvidos para a distribuição generalizada dos preservativos e a promoção do seu uso têm sido positivos. As iniciativas e acções de prevenção devem alcançar um número elevado de indivíduos dos vários grupos a que se destinam. $\mathrm{O}$ facto de uma minoria dos participantes referir ter sido abrangida por campanhas de prevenção leva a reflectir sobre a percepção dos indivíduos face ao conceito de campanha de prevenção e a questionar se uma campanha ou instrumento de prevenção deve ser entendido como tal pela população-alvo, ou simplesmente ser funcional e eficiente. Este aspecto torna-se essencial na monitorização e avaliação de campanhas, processos que são fundamentais para a adequação e eficácia dos esforços desenvolvidos para promover o acesso aos serviços de prevenção.

Mais de dois terços dos participantes reportaram já ter efectuado o teste do VIH. Este é um resultado positivo na medida em que, como vários estudos demonstram, o conhecimento do estatuto serológico positivo para o VIH pode resultar numa diminuição significativa de comportamentos sexuais de risco (Marks et al., 2005; Schwarcz et al., 2006). Os dados da UNAIDS, de 2010, referentes a Portugal estimam que $17.5 \%$ da população nacional com idade entre 15 e 49 anos realizou o teste do VIH no último ano. Apesar de as idades não serem concordantes e não se poder fazer uma comparação linear, verifica-se que a proporção de realização do teste reportada pelos homens gays no presente estudo é muito superior (UNAIDS, 2010b). A taxa de realização do teste na população inquirida pode significar que esta está consciencializada para questões relacionadas com a infecção pelo VIH. Efectivamente, neste estudo os participantes revelaram um elevado nível de conhecimento sobre o facto de o teste VIH poder ser efectuado de forma gratuita e confidencial. No entanto, num estudo online europeu sobre homossexualidade, comportamento homossexual e infecções sexualmente transmissíveis entre homens que têm sexo com homens, os dados referentes a Portugal indicam que $45.9 \%$ dos participantes realizaram o teste no último ano, proporção inferior à observada no presente estudo (EMIS, 2010). O método de amostragem utilizado pode em parte explicar esta discrepância. $O$ facto de neste estudo os participantes terem sido recrutados com base nas redes sociais e contactos das organizações comunitárias parceiras pode ter tido impacto na composição da amostra ao potencialmente reunir indivíduos com maior acesso a informação e prevenção, o que pode ter produzido vieses nos resultados obtidos, nomeadamente uma sobrestimação no que respeita à realização do teste VIH.

De acordo com os resultados obtidos, um grupo de participantes (cerca de $12 \%$ ) reportou nunca ter feito o teste do VIH, o que reforça a necessidade de desenvolver esforços contínuos para a consciencialização da importância de realizar o teste e de conhecer o estatuto serológico, dado o papel determinante do diagnóstico precoce no acesso atempado a tratamentos e no aumento de 
comportamentos de prevenção da transmissão (Marks et al., 2006; Quinn et al., 2000). Os dados apontam também no sentido de que é importante reconhecer que as questões da prevenção da infecção pelo VIH são extremamente complexas e influenciadas por uma multiplicidade de factores, o que torna pertinente o desenvolvimento de estratégias integradas e abrangentes atendendo às especificidades das diversas populações.

Os resultados sobre a escolha do local de realização do teste estar relacionada com o nível socioeconómico deixa em aberto se a decisão se baseia na comodidade, confidencialidade ou qualidade do atendimento, entre outros factores. No entanto, estudos indicam que os serviços que atendem homens gays e outros homens que têm sexo com homens em questões relacionadas com o VIH, de uma forma geral, não valorizam a necessidade de disponibilizar informação específica sobre práticas preventivas no contexto das relações sexuais entre homens e sobre a frequência do rastreio (Johnson et al., 2009; Makadon et al., 2006; Mimiaga et al., 2007; Mimiaga et al., 2009), estabelecida para diferentes populações em orientações internacionalmente reconhecidas (CDC, 2010).

Os resultados revelam ainda que os participantes com nacionalidade estrangeira mais frequentemente efectuaram o teste numa $\mathrm{ONG}$, o que indica que as associações e organizações na comunidade são fundamentais para aceder a grupos minoritários, como as populações LGBT imigrantes e actuar ao nível da prevenção e promoção da realização do teste nestes grupos.

Os dados apresentados devem ser analisados tendo em conta algumas limitações inerentes ao estudo. No que respeita ao método de amostragem utilizado, o processo de bola de neve leva a que a composição da amostra seja fortemente influenciada pela escolha dos participantes iniciais. O recrutamento não-aleatório dos participantes através das suas redes sociais pode resultar num enviesamento da amostra ao reunir inquiridos com características sociodemográficas e comportamentais semelhantes. Efectivamente, a amostra deste estudo caracteriza-se, de forma geral, por ser jovem e ter elevados níveis de escolaridade e rendimentos. Também o recrutamento dos participantes iniciais com base na rede de contactos das organizações comunitárias pode ter contribuído para um enviesamento na composição da amostra, como já referido num ponto anterior da discussão. No entanto, este método de amostragem tem sido reconhecido como eficaz no alcance de populações de difícil acesso (Faugier \& Sargeant, 1997; Morrison, 1988), o que é visível no elevado número de homens gays que participaram neste estudo. Por outro lado, a recolha de dados decorreu em diversas regiões do país no sentido de aceder a uma maior diversidade de participantes. Outra limitação prende-se com o facto de a aplicação dos questionários ter sido realizada por inquiridores. Algumas implicações podem advir da utilização 
desta técnica, nomeadamente relacionadas com o facto de o questionário incidir sobre questões íntimas e sensíveis e de a sua aplicação por um inquiridor poder ser factor de inibição e condicionamento das respostas, em detrimento da resposta aos questionários por autopreenchimento. $\mathrm{O}$ treino dos inquiridores, em que nomeadamente se instruiu para não aplicarem questionários a pessoas que conhecessem pessoalmente, e os procedimentos de recolha de dados definidos, como a garantia do anonimato dos participantes e da confidencialidade da sua participação, contribuíram para minimizar potenciais constrangimentos associados à utilização desta técnica. Em contrapartida, a aplicação do questionário por inquiridores permitiu garantir a uniformidade de procedimentos do processo de recolha de dados (por exemplo, ao nível da leitura e interpretação das perguntas), maximizar o número de perguntas respondidas (e evitar as "não respostas") garantindo assim uma maior qualidade da informação recolhida, e assegurar a inclusão de participantes com menores competências de compreensão escrita (por exemplo, com baixa escolaridade ou estrangeiros).

Apesar das limitações, este é um dos poucos estudos realizados em Portugal com homens gays e com a inclusão de um elevado número de participantes. Este estudo realça um conjunto de temáticas que deverão ser aprofundadas em futuras investigações, como as questões relacionadas com a procura e utilização de serviços de saúde no contexto do VIH/sida por parte das populações LGBT, nomeadamente as percepções e experiências relativas aos serviços e profissionais de saúde, as potenciais barreiras na sua utilização e a frequência e motivos de realização do teste atendendo às diferenças demográficas, socioeconómicas e culturais nesta população.

Os serviços de saúde desempenham um papel crucial ao nível da informação, prevenção e promoção do teste VIH, em especial em grupos mais vulneráveis (WHO, 2009). Neste sentido é importante adequar a prestação de cuidados de saúde no contexto da diversidade sexual bem como promover o acesso e a utilização dos serviços por parte das populações LGBT, em especial os grupos em maior desvantagem social e económica, trabalhando em proximidade com as comunidades e conhecendo as suas necessidades de saúde específicas (Mimiaga et al., 2009).

\section{Referências}

AMFAR. (2008). MSM, HIV, and the road to universal access - How far have we come? Special Report. Nova Iorque: AMFAR.

AMFAR. (2006, Junho). HIV prevention for men who have sex with men. Issue Brief, 4. Recuperado em 18 Setembro, 2011, de http://www.amfar. 
org/uploadedFiles/In_the_Community/Publications/HIV\%20Prevention $\% 20$ for \%20MSM.pdf

Bakker, F., Sandfort, T., Vanwesenbeeck, I., Lindert, H., \& Westert, G. (2006). Do homosexual persons use health care services more frequently than heterosexual persons: Findings from a Dutch population survey. Social Science \& Medicine, 63(8), 2022-2030.

Baral, S., et al. (2007). Elevated risk for HIV infection among men who have sex with men in low-and middle-income countries 2000-2006: A systematic review. PLoS Medicine, 4(12), e339.

Chen, S.Y., Gibson, S., Katz, M.H., Klausner, J.D., Dilley, J.W., Schwarcz, S.K., et al. (2002). Continuing increases in sexual risk behavior and sexually transmitted diseases among men who have sex with men: San Francisco, Calif, 1999-2001, USA. American Journal of Public Health, 92, 1387-1388.

Clark, M.E., Landers, S., Linde, R., \& Sperber, J. (2001). The GLBT health access project: A state-funded effort to improve access to care. American Journal of Public Health, 91(6), 895-896.

Dowshen, N., Binns, H., \& Garofalo, R. (2009). Experiences of HIV-related stigma among young men who have sex with men. AIDS Patient Care and STDS, 23(5): 371-376.

ECDC/WHO. (2010). HIV surveillance in Europe 2009. Stockholm: European Centre for Disease Prevention and Control.

Eliason, M.J., \& Schope, R. (2001). Does “don't ask don't tell” apply to health care? Lesbian, gay, and bisexual people's disclosure to health care providers. Journal of Gay and Lesbian Medical Association, 5, 125-134.

EMIS (2010). The European MSM Internet Survey (EMIS) - $1^{\circ}$ Relatório comunitário. Recuperado em 18 Setembro, 2011, de http://www.emis-project.eu/sites/default/files/public/publications/EMIS_community1_Portugue se_0.pdf

Faugier, J., \& Sargeant, M. (1997). Sampling hard to reach populations. Journal of Advanced Nursing, 26, 790-797.

Hatzenbuehler, M.L., O’Cleirigh, C., Mayer, K.H., \& Mimiaga, M.J. (2011). Prospective associations between HIV-related stigma, transmission risk behaviors, and adverse mental health outcomes in men who have sex with men. Annals of Behavioral Medicine, 42, 227-234.

Heymer, K.J., \& Wilson, D.P. (2011). Treatment for prevention of HIV transmission in a localized epidemic: The case for South Australia. Sexual Health, 8(3), 280$-294$.

INSA. (2011). Infecção VIH/SIDA: A situação em Portugal a 31 de Dezembro de 2010. Lisboa: INSA.

Johnson, C.V., Mimiaga, M.J., Reisner, S.L., et al. (2009). Health care access and sexually transmitted infection screening frequency among at-risk Massachusetts men who have sex with men. American Journal of Public Health, 99(1), S187-192. 
Koblin, B.A., Husnik, M.J., Colfax, G., Huang, Y., Madison, M., Mayer, K., Barresi, P.J., Coates, T.J., Chesney, M.A., \& Buchbinder, S. (2006). Risk factors for HIV infection among men who have sex with men. AIDS, 20, 731-739.

Likatavicius, G., Klavs, I., Devaux, I., Alix, J., \& Nardone, A. (2008). An increase in newly diagnosed HIV cases reported among men who have sex with men in Europe, 2000-6: Implications for a European public health strategy. Sexually Transmitted Infections, 84(6), 499-505.

Makadon, H.J., Mayer, K.H., \& Garofalo, R. (2006). Optimizing primary care for men who have sex with men. Journal of the American Medical Association, 296, 2362-2365.

Marks, G., Crepaz, N., \& Janssen, R.S. (2006). Estimating sexual transmission of HIV from persons aware and unaware that they are infected with the virus in the USA. AIDS, 20, 1447-1450.

Marks, G., Crepaz, N., Senterfitt, J., \& Janssen, S. (2005). Meta-analysis of high-risk sexual behavior in persons aware and unaware they are infected with HIV in the United States: Implications for HIV prevention programs. Journal of Acquired Immune Deficiency Syndromes, 39(4), 446-453.

Mayer, K.H., Bradford. J.B., Makadon. H.J., Stall. R., Goldhammer. H., \& Landers, S. (2008). Sexual and gender minority health: what we know and what needs to be done. American Journal of Public Health, 98(6), 989-995.

Melendez, R.M., \& Pinto, R.M. (2009). HIV prevention and primary care for transgender women in a community-based clinic. Journal of the Association of Nurses in AIDS Care, 20(5), 387-397.

Meyer, I.H. (2003). Prejudice, social stress, and mental health in lesbian, gay, and bisexual populations: Conceptual issues and research evidence. Psychological Bulletin, 129(5), 674-697.

Mimiaga, M.J., Goldhammer, H., Belanoff, C., Tetu, A.M., \& Mayer, K.H. (2007). Men who have sex with men: Perceptions about sexual risk, HIV and sexually transmitted disease testing, and provider communication. Sexually Transmitted Diseases, 34, 113-119.

Mimiaga, M.J., Reisner, S.L., Bland, S., Skeer, M., Cranston, K., Isenberg, D., Vega, B.A., \& Mayer, K.H. (2009). Health system and personal barriers resulting in decreased utilization of HIV and STD testing services among at-risk black men who have sex with men in Massachusetts. AIDS Patient Care and STDS, 23(10), 825-835.

Morrisson, V. (1988). Observation and snowballing: Useful tools for research into illicit drug use? Social Pharmacology, 2(3), 247-271.

Ordem dos Psicólogos Portugueses. (2011). Regulamento n. ${ }^{\circ}$ 258/2011. Diário da República n. ${ }^{\circ}$ 78, Série II de 20 de Abril de 2011.

Ottosson, D. (2010). State-sponsored homophobia. A world survey of laws prohibiting same sex activity between consenting adults. Recuperado em 17 Setembro, 2011, de http://old.ilga.org/Statehomophobia/ILGA_State_Sponsored_Homo phobia_2010.pdf

Quinn, T.C., Wawer, M.J., Sewankambo, N., et al. (2000). Viral load and heterosexual transmission of human immunodeficiency virus type 1. Rakai Project Study Group. The New England Journal of Medicine, 342, 921-929. 
Reisner, S.L., Mimiaga, M.J., Skeer, M., Bright, D., Cranston, K., Isenberg, D., Bland, S., Barker, T.A., \& Mayer, K.H. (2009). Clinically significant depressive symptoms as a risk factor for HIV infection among black MSM in Massachusetts. AIDS and Behavior, 13, 798-810.

Sadler, G., Lee, H., Lim, R., \& Fullerton, J. (2010). Recruitment of hard-to-reach population subgroups via adaptations of the snowball sampling strategy. Nursing and Health Sciences, 12, 369-374.

Schuster, M., Collins, R., Cunningham, W., Morton, S., Zierler, S., Wong, M., Tu, W., \& Kanouse, D. (2005). Perceived discrimination in clinical care in a nationally representative sample of HIV-infected adults receiving health care. Journal of General Internal Medicine, 20, 807-813.

Schwarcz, S., Hsu, L., Dilley, J., Loeb, L., Nelson, K., \& Boyd, S. (2006). Late diagnosis of HIV infection: trends, prevalence, and characteristics of persons whose HIV diagnosis occurred within 12 months of developing AIDS. Journal of Acquired Immune Deficiency Syndromes, 43(4), 491-494.

Stall, R., Mills, T., Williamson, J., Hart, T., Greenwood, G., Paul, J., Pollack, L., Binson, D., Osmond, D., \& Catania, J. (2003). Association of co-occurring psychosocial health problems and increased vulnerability to HIV/AIDS among urban men who have sex with men. American Journal of Public Health, 93(6), 939-942.

Stein, G.L., \& Bonuck, K.A. (2001). Physician-patient relationships among the lesbian and gay community. Journal of Gay and Lesbian Medical Association, 5, 87-93.

UNAIDS. (2009a). UNAIDS Action Framework: Universal Access for Men who have Sex with Men and Transgender People. Geneva: UNAIDS.

UNAIDS. (2009b). Monitoring the Declaration of Commitmenton HIV/AIDS: Guidelines on Construction of Core Indicators: 2010 Reporting. Geneva: UNAIDS.

UNAIDS. (2010a). Global report: UNAIDS report on the global AIDS epidemic. Geneva: UNAIDS.

UNAIDS. (2010b). Country Progress Reports submitted to UNAIDS in 2010 for the UNGASS reporting process. Recuperado em 18 Setembro, 2011, de http:/www.unaids.org/en/regionscountries/countries/portugal/

Valdiserri, R. (2002). HIV/AIDS stigma: An impediment to public health. American Journal of Public Health, 92, 341-342.

van de Laar, M.J. (2009). HIV/AIDS and other STI in men who have sex with men A continuous challenge for public health. Eurosurveillance, 14(47), 19423. Recuperado em 17 Setembro, 2011, de http://www.eurosurveillance.org/ ViewArticle.aspx?ArticleId=19423

van Griensvena, F., van Wijngaardenc, J.W., Barald, S., \& Grulich, A. (2009). The global epidemic of HIV infection among men who have sex with men. Current Opinion in HIV and AIDS, 4, 300-307.

Wasserman, S., Pattison, P., \& Steinley, D. (2005). Encyclopedia of statistics in behavioral science. Hoboken, NJ: John Wiley \& Sons. 
WHO. (2007). Everybody business: Strengthening health systems to improve health outcomes - WHO's framework for action. Geneva: WHO.

WHO. (2009). Prevention and treatment of HIV and other sexually transmitted infections among men who have sex with men and transgender populations: Report of a technical consultation, 15-17 September 2008. Geneva: WHO.

WHO. (2011). Guidelines: prevention and treatment of HIV and other sexually transmitted infections among men who have sex with men and transgender people: recommendations for a public health approach 2011. Geneva: WHO.

Wolitski, R.J., \& Fenton, K.A. (2011). Sexual health, HIV, and sexually transmitted infections among gay, bisexual, and other-S17.

Young, R.M., \& Meyer, I.H. (2005). The trouble with "MSM" and "WSW": Erasure of the sexual-minority person in public health discourse. American Journal of Public Health, 95, 1144-1149. 